\title{
Effects of oral Lactobacillus administration on antioxidant activities and CD4+CD25+forkhead box P3 (FoxP3)+ T cells in NZB/W F1 mice
}

\author{
Bor-Show Tzang ${ }^{1,2,3,4}$, Chung-Hsien Liu ${ }^{5}$, Kuo-Ching Hsu ${ }^{1}$, Yi-Hsing Chen ${ }^{6,7}$, Chih-Yang Huang ${ }^{8,9,10_{\dagger}}$ \\ and Tsai-Ching $\mathrm{Hsu}^{1,2,3 *}$ \\ ${ }^{1}$ Institute of Biochemistry, Microbiology and Immunology, Chung Shan Medical University, Taichung 402, Taiwan, ROC \\ ${ }^{2}$ Immunology Research Center, Chung Shan Medical University, Taichung 402, Taiwan, ROC \\ ${ }^{3}$ Clinical Laboratory, Chung Shan Medical University Hospital, Taichung 402, Taiwan, ROC \\ ${ }^{4}$ Department of Biochemistry, School of Medicine, Chung Shan Medical University, Taichung 402, Taiwan, ROC \\ ${ }^{5}$ Department of Obstetrics and Gynecology, Chung Shan Medical University and Chung Shan Medical University Hospital, \\ Taichung 402, Taiwan, ROC \\ ${ }^{6}$ Research and Development Department, GenMont Biotech Incorporation, Tainan 741, Taiwan, ROC \\ ${ }^{7}$ Institute of Biomedical Science and RongHsing Research Center for Translational Medicine, National Chung-Hsing \\ University, Taichung 402, Taiwan, ROC \\ ${ }^{8}$ Graduate Institute of Basic Medical Science, China Medical University, Taichung 404, Taiwan, ROC \\ ${ }^{9}$ Graduate Institute of Chinese Medical Science, China Medical University, Taichung 404, Taiwan, ROC \\ ${ }^{10}$ Department of Health and Nutrition Biotechnology, Asia University, Taichung 413, Taiwan, ROC
}

(Submitted 3 May 2017 - Final revision received 17 July 2017 - Accepted 24 July 2017)

\section{Abstract}

Systemic lupus erythematosus (SLE) is an autoimmune disease that is characterised by a dysregulation of the immune system, which causes inflammation responses, excessive oxidative stress and a reduction in the number of cluster of differentiation (CD) $4+\mathrm{CD} 25+$ forkhead box P3 (FoxP3)+ T cells. Supplementation with certain Lactobacillus strains has been suggested to be beneficial in the comprehensive treatment of SLE. However, little is known about the effect and mechanism of certain Lactobacillus strains on SLE. To investigate the effects of Lactobacillus on SLE, NZB/W F1 mice were orally gavaged with Lactobacillus paracasei GMNL-32 (GMNL-32), Lactobacillus reuteri GMNL-89 (GMNL-89) and L. reuteri GMNL-263 (GMNL-263). Supplementation with GMNL-32, GMNL-89 and GMNL-263 significantly increased antioxidant activity, reduced IL- 6 and TNF- $\alpha$ levels and significantly decreased the toll-like receptors/myeloid differentiation primary response gene 88 signalling in NZB/W F1 mice. Notably, supplementation with GMNL-263, but not GMNL-32 and GMNL-89, in NZB/W F1 mice significantly increased the differentiation of CD4+CD25+FoxP3+ T cells. These findings reveal beneficial effects of GMNL-32, GMNL-89 and GMNL-263 on NZB/W F1 mice and suggest that these specific Lactobacillus strains can be used as part of a comprehensive treatment of SLE patients.

Key words: Systemic lupus erythematosus: Comprehensive treatment: Lactobacillus paracasei GMNL-32: Lactobacillus reuteri GMNL-89: Lactobacillus reuteri GMNL-263

Various studies have shown that elevated oxidative stress and decreased antioxidant activities are critical in the pathogenesis of systemic lupus erythematosus (SLE) ${ }^{(1-3)}$. Excessive oxidative stress damages lipids, proteins and $\mathrm{DNA}^{(4,5)}$, and elicits autoantibodies in SLE patients, such as antibodies against oxidatively modified DNA and $\mathrm{LDL}^{(6,7)}$. Cytokines are also known to play direct roles in the disease pathogenesis of SLE ${ }^{(8)}$. SLE is characterised by a dysregulation of the immune system, which causes inflammation in multiple organs with diverse clinical manifestations ${ }^{(9)}$. Levels of IL- 6 and TNF- $\alpha$ in serum are elevated in both lupus animal models and SLE patients. Studies of multiple animal SLE models have identified the critical role of the IL-6 pathway in SLE ${ }^{(10-13)}$. Indeed, evidence has correlated serum IL-6 level with disease activity or anti-double stranded

Abbreviations: DPPH, 1,1-diphenyl-2-picryl-hydrazyl; FoxP3, forkhead box P3; GMNL-32, Lactobacillus paracasei GMNL-32; GMNL-89, Lactobacillus reuteri GMNL-89; GMNL-263, Lactobacillus reuteri GMNL-263; MDA, malondialdehyde; MyD88, myeloid differentiation primary response gene 88; PI3K, phosphoinositide 3-kinase; SLE, systemic lupus erythematosus; TLR, toll-like receptor.

* Corresponding author: Dr T.-C. Hsu, fax +886 42324 8172, email htc@csmu.edu.tw

$\dagger$ Equal contribution as corresponding author. 
DNA levels in SLE patients ${ }^{(14)}$. Similar to IL-6 expression, TNF- $\alpha$ expression is also increased in lupus-prone animal models and SLE patients. Elevated TNF- $\alpha$ level has been correlated with disease activity in both lupus-prone animal models and SLE patients ${ }^{(15-18)}$.

Our understanding of the beneficial roles of certain probiotics in sustaining human health has increased substantially in recent years ${ }^{(19-21)}$. Increasing scientific literature has indicated that supplementation with certain probiotics is a beneficial part of a comprehensive treatment of inflammatory disease. These specific probiotic strains or their components bind toll-like receptors (TLR) or Nod-like receptors to modulate directly the activity of various inflammatory signalling pathways $^{(22,23)}$. These effects have been demonstrated in both animal models and clinical studies ${ }^{(24,25)}$. Particular probiotics also participate in the differentiation and functions of immune cells that are associated with an inflammatory response, including dendritic cells and $\mathrm{T}$ cells ${ }^{(22,23)}$. Notably, recent studies have found that certain Lactobacillus strains promote the production of anti-inflammatory cytokines such as IL-10 and transforming growth factor- $\beta$, to treat autoimmune diseases such as $\operatorname{SLE}^{(19,26,27)}$

The Lactobacillus genus is a major part of the lactic acid bacteria group, and over 170 species of Lactobacillus genus have been reported ${ }^{(28)}$ Although some species of Lactobacillus genus exist in normal human gastrointestinal and vaginal flora, they can also be occasional pathogens in humans ${ }^{(29)}$. Indeed, we also identified the beneficial effects of Lactobacillus reuteri GMNL-263 (GMNL-263) in sustaining heart function and attenuating hepatic and cardiac fibrosis in animal models ${ }^{(30,31)}$. Recently, evidence has shown an imbalance of gastrointestinal microflora in the pathogenesis of $\mathrm{SLE}^{(32)}$. Supplementation with certain Lactobacillus strains has been suggested to be a beneficial part of comprehensive treatments of autoimmune diseases such as $\operatorname{SLE}^{(19,26,27)}$. Notably, our recent study also reported the beneficial effects of Lactobacillus paracasei GMNL-32 (GMNL-32) and L. reuteri GMNL-89 (GMNL-89) and L. reuteri GMNL-263 on hepatic inflammation and apoptosis in NZB/W F1 mice ${ }^{(33)}$. To clarify further the beneficial effects of GMNL-32, GMNL-89 and GMNL-263 on SLE, NZB/W F1 mice were administered GMNL-32, GMNL-89 or GMNL-263, and the antioxidant activities, expressions of TLR, proinflammatory cytokines and CD4+CD25+forkhead box P3 (FoxP3)+ T cells were analysed.

\section{Methods \\ Preparation of Lactobacillus strains}

GMNL-32, GMNL-89 and GMNL-263 were obtained from GenMont Biotech, Inc., and the doses of these Lactobacillus strains $\left(10^{9}\right.$ colony-forming units (CFU)/mouse per $\mathrm{d}$ ) that were used in this study were based on our previous publications ${ }^{(30,31,33,34)}$. The catalogue numbers at the Bioresource Collection and Research Center (BCRC) in Taiwan are 'BCRC 910220', 'BCRC 910340' and 'BCRC 910452', respectively. Powders of three Lactobacillus strains were prepared in PBS for use in the oral gavage treatment of mice.

\section{Animal and treatments}

This study was approved by the Institutional Animal Care and Use Committee at Chung Shan Medical University (approval no. 84). A total of thirty-two female NZB/W F1 mice aged 6 weeks were purchased from Jackson Laboratory. All animals were kept in a $12 \mathrm{~h}$ light $-12 \mathrm{~h}$ dark cycle and ambient temperature was maintained at $25^{\circ} \mathrm{C}$. Animals had free access to water and standard laboratory chow (LabDiet 5001; PMI Nutrition International Inc.). Animal welfare was maintained and experimental procedures were performed according to the National Institutes of Health Guide for the Care and Use of Laboratory Animals. At an age of 8 weeks, animals were randomly divided into four groups (each of eight mice) - control, GMNL-32, GMNL-89 and GMNL-263 groups - which are treated with PBS, $10^{9} \mathrm{CFU} /$ mouse per $\mathrm{d}$ of GMNL-32, GMNL-89 and GMNL-263, respectively, by oral gavage. The experimental period was 12 weeks long and the mice were killed by asphyxiation using $\mathrm{CO}_{2}$ at an age of 20 weeks. Heart blood and liver tissues were collected and stored at $-80^{\circ} \mathrm{C}$ until analysis.

\section{ELISA}

The levels of TNF- $\alpha$ and IL- 6 were detected with TNF- $\alpha$ and IL- 6 ELISA kits (Thermo Fisher Scientific) according to the manufacturer's instructions.

\section{Serum glutathione levels}

Serum glutathione levels were measured as described elsewhere ${ }^{(35)}$. Concentrations of reduced glutathione GSH were measured using a GSH assay kit (Chemicon Inc.) according to the manufacturer's instructions.

\section{1,1-Diphenyl-2-picryl-hydrazyl assay}

The radical scavenging activity of 1,1-diphenyl-2-picryl-hydrazyl (DPPH) was measured as described in our previous work ${ }^{(35)}$. In brief, a solution of $180 \mu \mathrm{l}$ of $0 \cdot 1 \mathrm{~mm}$ DPPH solution in ethanol was gently mixed with $20 \mu \mathrm{l}$ of serum sample in ethanol. The DPPH absorption was measured at $517 \mathrm{~nm}$ using a ninety-six-well fluorometric plate reader and the DPPH radical scavenging activity was expressed as percentage inhibition relative to ethanol.

\section{Thiobarbituric acid reductase assay}

Lipid peroxidation was measured using a thiobarbituric acid reductase (TBAR) assay, as described elsewhere ${ }^{(35)}$. In brief, to make a $10 \%$ homogenate, $200 \mathrm{mg}$ of serum was added to $2 \mathrm{ml}$ of $1.15 \% \mathrm{KCl}$ and mixed using a homogeniser (Knotes Glass). A $3-\mathrm{ml}$ volume of $1 \%$ phosphoric acid and $1 \mathrm{ml}$ of $0.6 \%$ TBA solution were added to $0.5 \mathrm{ml}$ of $10 \%$ homogenate. The mixture was left in a boiling water bath for $45 \mathrm{~min}$. After cooling, $4 \mathrm{ml}$ of n-butanol was added and the mixture was mixed vigorously. The butanol phase was separated by centrifugation at $500 \boldsymbol{g}$, and absorbance was measured spectrophotometrically at $535 \mathrm{~nm}$. The difference was used as the TBAR value, which was calculated in $\mathrm{nmol} / \mathrm{g}$ of homogenate. 

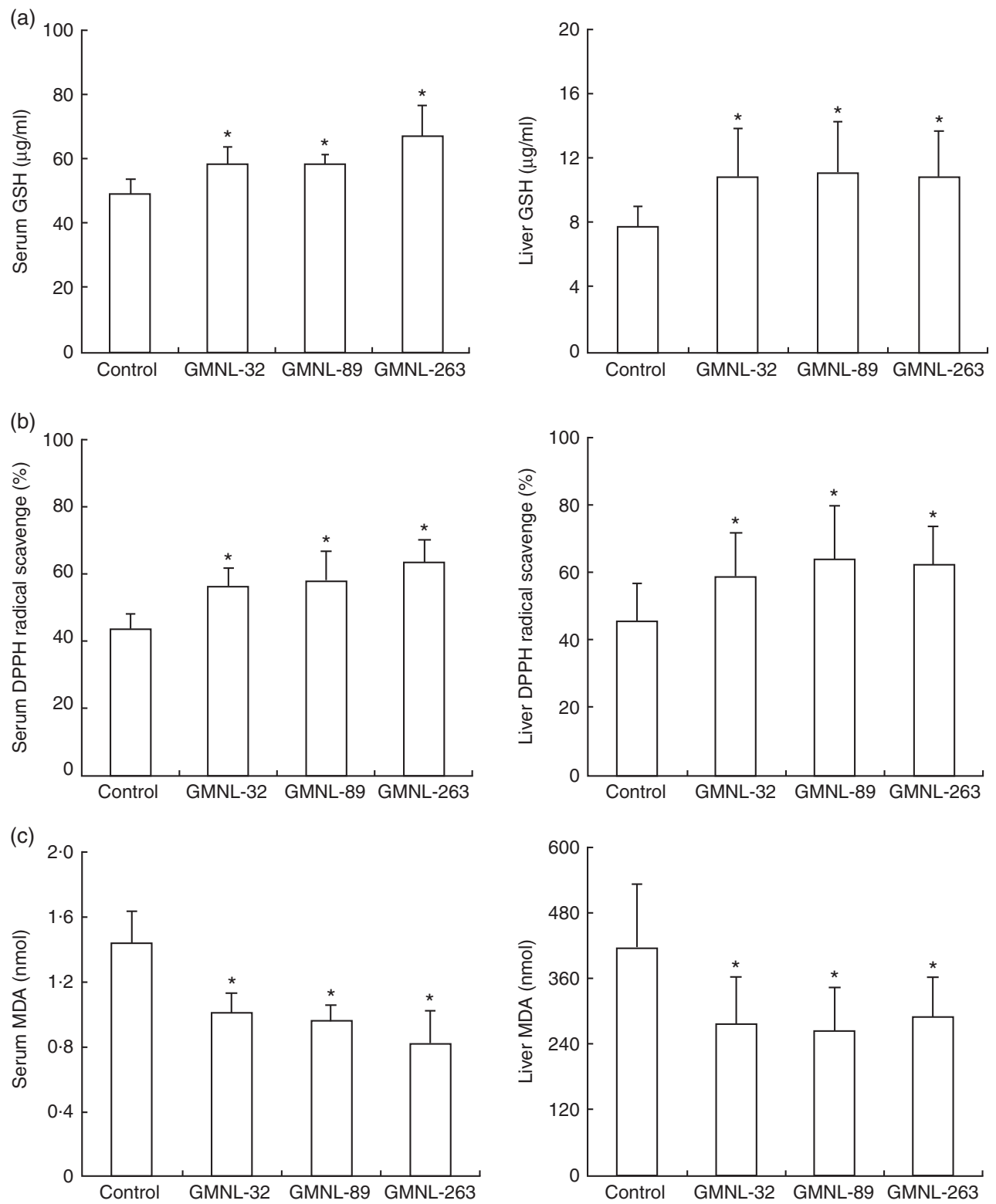

Fig. 1. Effects of Lactobacillus paracasei GMNL-32 (GMNL-32), Lactobacillus reuteri GMNL-89 (GMNL-89) and L. reuteri GMNL-263 (GMNL-263) on serum and liver (a) GSH, (b) 1,1-diphenyl-2-picryl-hydrazyl (DPPH) and (c) malondialdehyde (MDA) levels in NZB/W F1 mice. Values are means ( $n 8$ animals/group), and standard deviations represented by vertical bars. Similar results were observed in three repeated experiments. Mean value was significantly different from that of the control group: * $P<0.05$

\section{Preparation of tissue extract}

All procedures were performed at $4^{\circ} \mathrm{C}$ in a cold room. The liver samples obtained from NZB/W F1 mice were homogenised in $600 \mu \mathrm{l}$ of PRO-PREPTM solution (iNtRON Biotech) by thirty strokes using a Dounce Homogenizer (Knotes Glass). The homogenates were centrifuged at $13000 \mathrm{rpm}$ for $10 \mathrm{~min}$ at $4^{\circ} \mathrm{C}$ and the supernatant was then stored at $-80^{\circ} \mathrm{C}$ until use.

\section{Immunoblotting}

Liver samples were separated in $10 \%$ or $12.5 \%$ SDS-PAGE and electrophoretically transferred to nitrocellulose membrane
(Amersham Biosciences) described elsewhere ${ }^{(35)}$. After blocking with 5\% non-fat dry milk in PBS, antibodies against TLR- $4,5,7$ and 9, myeloid differentiation primary response gene 88 (MyD88), phosphorylated (p)-phosphoinositide 3-kinase (PI3K), PI3K, p-protein kinase B (AKT) and AKT (Santa Cruz Biotechnology) and $\beta$-actin (EMD Millipore) were diluted in PBS with $2.5 \%$ bovine serum albumin (BSA) and incubated for $1.5 \mathrm{~h}$ with gentle agitation at room temperature. The membranes were washed twice with PBS-Tween for $1 \mathrm{~h}$ and secondary antibody conjugated with horseradish peroxidase (HRP) (Santa Cruz Biotechnology) was added. Immobilon Western HRP Chemiluminescent Substrate (EMD Millipore) was then used to detect the antigen-antibody complexes. The blots were scanned 
and semiquantified by densitometry (Alpha-Imager 2200; ProteinSimple).

\section{Detection of T-cell subpopulations}

Mice were killed at the age of 20 weeks and their spleens were harvested and placed in cold RPMI-1640 medium that was supplemented with $10 \%$ fetal bovine serum (Invitrogen), $10 \mathrm{mg} / \mathrm{ml}$ gentamycin, $2 \mathrm{~mm}$ L-glutamine and $0.1 \mathrm{~mm}$ 2-mercaptoethanol. Erythrocytes were lysed in BD FACS $^{\text {TM }}$ lysing solution (BD Biosciences) on ice, according to the manufacturer's instructions. Splenocytes were stained with optimal concentrations of fluorochrome-conjugated mAbs ( $10^{6}$ cells in $200 \mu$ l of PBS, $1 \%$ BSA and $0 \cdot 1 \%$ sodium azide) and fixed with $1 \%$ paraformaldehyde. Monoclonal antibodies against cluster of differentiation (CD) 3 conjugated with fluorescein isothiocyanate (FITC), CD4 conjugated with FITC or R-phycoerythrin (PE), CD8 conjugated with FITC and CD25 conjugated with PE were used to analyse T-cell markers (BD Pharmingen). To purify CD4+CD25+ regulatory T cells, a CD4 +CD25+ regulatory T-Cell Isolation Kit was used according to the manufacturer's instructions (Miltenyi Biotec). Samples were analysed using a FACSCalibur instrument (Becton Dickinson) and stored at $-80^{\circ} \mathrm{C}$ for immunoblotting assay.

\section{Detection of forkhead box P3 mRNA}

The mRNA expression of FoxP3 was measured as described elsewhere $^{(35)}$. Total cellular mRNA was extracted from $1 \times 10^{6}$ isolated CD4+CD25+ splenic cells using Dynabeads mRNA DIRECT $^{\mathrm{TM}}$ (Invitrogen) and reverse transcription was performed using SuperScript TM II RT (Invitrogen). Gene expression was quantified using real-time PCR with Assays-on-Demand TM gene expression products (FoxP3, hypoxanthine phosphoribosyltransferase (HPRT)) and the ABI/PRISM 7700 sequence detection system (Applied Biosystems Inc.). Experiments were performed and repeated three times. The relative expression of FoxP3 was obtained by dividing the FoxP3 gene by the HPRT expression.

\section{Statistical analysis}

Sample size was calculated using free sample size calculating software G*Power version 3.1.9.2 (Franz, Universitat Kiel). With a power of $80 \%, 0.05$ level of statistical significance and effect size of $0 \cdot 8$, the sample size for each test was calculated to be 8 . A total of thirty-two mice were randomly assigned into one of the four experimental groups. All values are expressed as means and standard deviations. The comparisons in GSH, DPPH and malondialdehyde (MDA) assay, IL-6 and TNF- $\alpha$ levels and immunoblot among groups were performed using GraphPad Prism 5 software (GraphPad Software, Inc.) by one-way ANOVA followed by Tukey's multiple comparisons test. $P<0.05$ was considered to indicate a statistically significant difference. The significant differences were stressed with symbols as shown in figures.

\section{Results}

Supplementation with Lactobacillus paracasei GMNL-32, Lactobacillus reuteri GMNL-89 and Lactobacillus reuteri GMNL-263 increases antioxidant activity and reduces IL-6 and TNF- $\alpha$ levels in NZB/W F1 mice

To investigate the effects of GMNL-32, GMNL-89 and GMNL-263 on antioxidant activities, the levels of GSH, DPPH and MDA in both serum and liver samples from NZB/W F1 mice were measured. Significantly higher serum and liver GSH and DPPH levels were detected in NZB/W F1 mice that had been treated with GMNL-32, GMNL-89 or GMNL-263, respectively, compared with mice that had been given a vehicle (control) (Fig. 1(a) and (b)). Significantly lower serum and liver MDA levels were found in NZB/W F1 mice that had been treated with GMNL-32, GMNL-89 or GMNL-263 compared with mice that had been given a vehicle (control) (Fig. 1(c)). Significantly lower serum IL-6 and TNF- $\alpha$ levels were detected in NZB/W F1 mice that had been treated with GMNL-32, GMNL-89 or GMNL-263 compared with mice that had been given a vehicle (control) (Fig. 2).
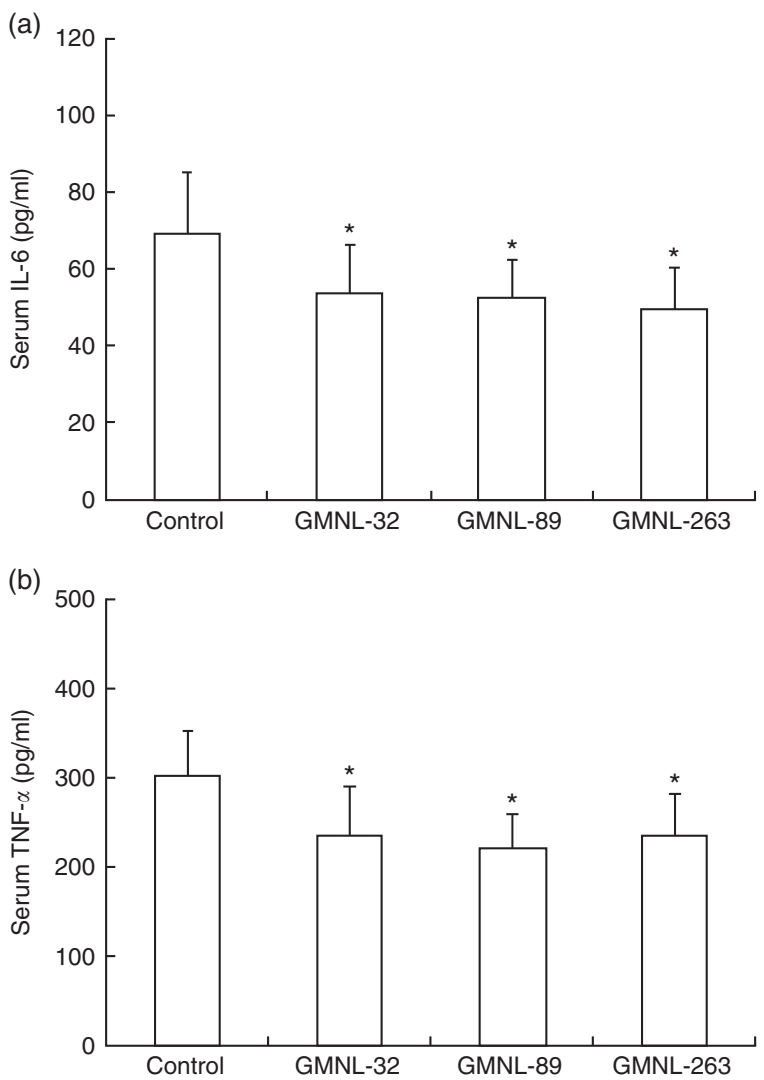

Fig. 2. Effects of Lactobacillus paracasei GMNL-32 (GMNL-32), Lactobacillus reuteri GMNL-89 (GMNL-89) and L. reuteri GMNL-263 (GMNL-263) on serum (a) IL-6 and (b) TNF-a expression in NZB/W F1 mice. Values are means ( $n 8$ animals/group), and standard deviations represented by vertical bars. Similar results were observed in three repeated experiments. Mean value was significantly different from that of the control group: * $P<0.05$. 
Supplementation with Lactobacillus paracasei GMNL-32, Lactobacillus reuteri GMNL-89 and Lactobacillus reuteri GMNL-263 ameliorates toll-like receptors/myeloid differentiation primary response gene 88 signalling in NZB/W F1 mice

To investigate the possible pathway by which Lactobacillus reduces IL- 6 and TNF- $\alpha$ levels in NZB/W F1 mice, TLR-4, TLR-5, TLR-7, TLR-9, MyD88, p-PI3K and p-AKT proteins were detected by immunoblotting. Significantly lower TLR-4, TLR-5, TLR-7 and TLR-9 expressions were detected in the livers of NZB/W F1 mice that had been treated with GMNL-32, GMNL-89 or GMNL-263 compared with mice that had been given a vehicle (control) (Fig. 3(a)). Fig. 3(b)-(e) present TLR-4, TLR-5, TLR-7 and TLR-9 levels relative to the $\beta$-actin level, respectively.

The level of MyD88, a downstream molecule of TLR-4, TLR-5, TLR-7 and TLR-9, was significantly lower in the livers of NZB/W F1 mice that had been treated with GMNL-32, GMNL-89 or
GMNL-263 than in the livers of mice that had been given a vehicle (control) (Fig. 4(a)). Fig. 4(a) presents MyD88 level relative to $\beta$-actin level. Moreover, levels of p-PI3K and p-AKT, the downstream molecules of MyD88, were significantly lower in the livers of NZB/W F1 mice that had been treated with GMNL-32, GMNL-89 or GMNL-263 than in the livers of mice that had been given a vehicle (control) (Fig. 4(b)). Fig. 4(b) presents p-PI3K level relative to PI3K level and p-AKT level relative to AKT level.

\section{Supplementation with Lactobacillus reuteri GMNL-263 increases the expression of $C D 4+C D 25+$ regulatory $T$ cell in NZB/W F1 mice}

To investigate further the immunoregulatory effects of GMNL-32, GMNL-89 and GMNL-263, especially on the proliferation of regulatory $\mathrm{T}$ cells, the expression of $\mathrm{CD} 4+\mathrm{CD} 25+$ regulatory
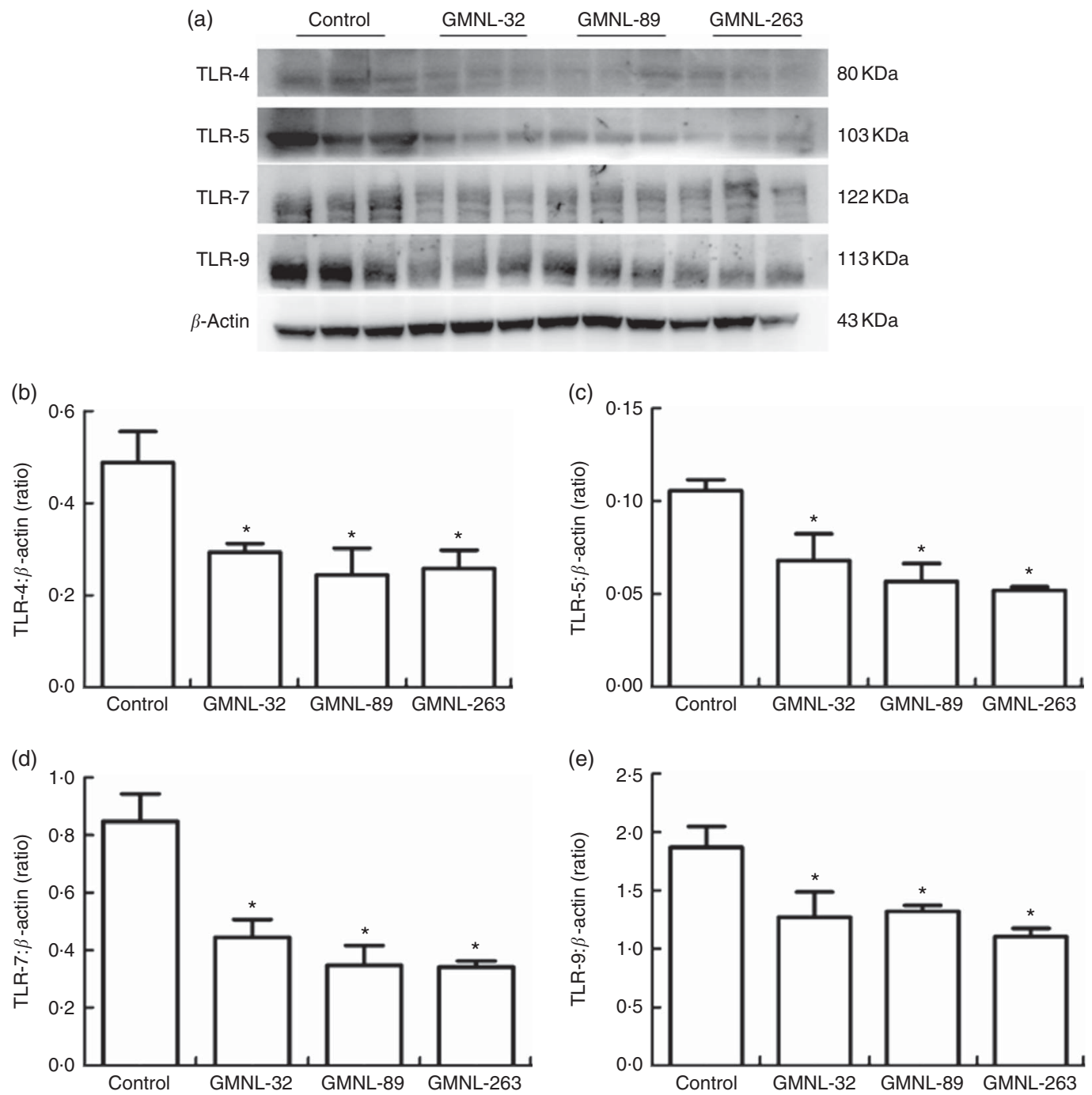

Fig. 3. Effects of Lactobacillus paracasei GMNL-32 (GMNL-32), Lactobacillus reuteri GMNL-89 (GMNL-89) and L. reuteri GMNL-263 (GMNL-263) on liver TLR expression. Liver lysates obtained from NZB/W F1 mice receiving vehicle (control) $(n 8)$, GMNL-32 ( $n 8)$, GMNL-89 $(n 8)$ or GMNL-263 ( $n 8$ ), respectively, were probed with antibodies against (a) toll-like receptors (TLR)-4, -5, -7 and -9. Bars represent the relative protein quantification of (b) TLR-4, (c) TLR-5, (d) TLR-7 and (e) TLR-9 on the basis of $\beta$-actin. Similar results were observed in three repeated experiments. Mean value was significantly different from that of the control group: ${ }^{*} P<0.05$. 

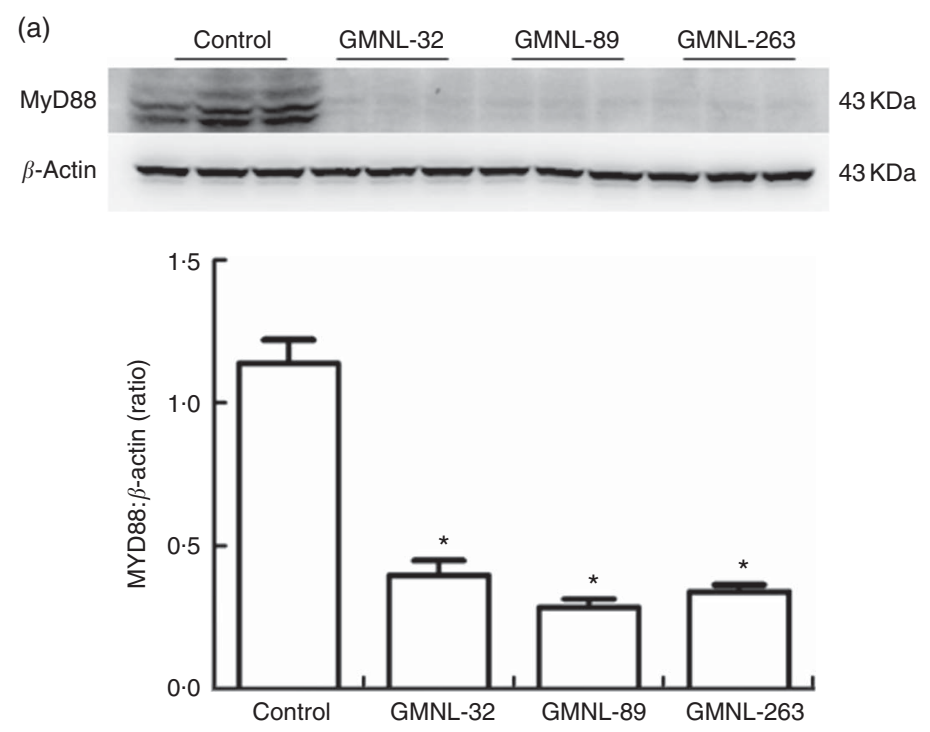

(b)
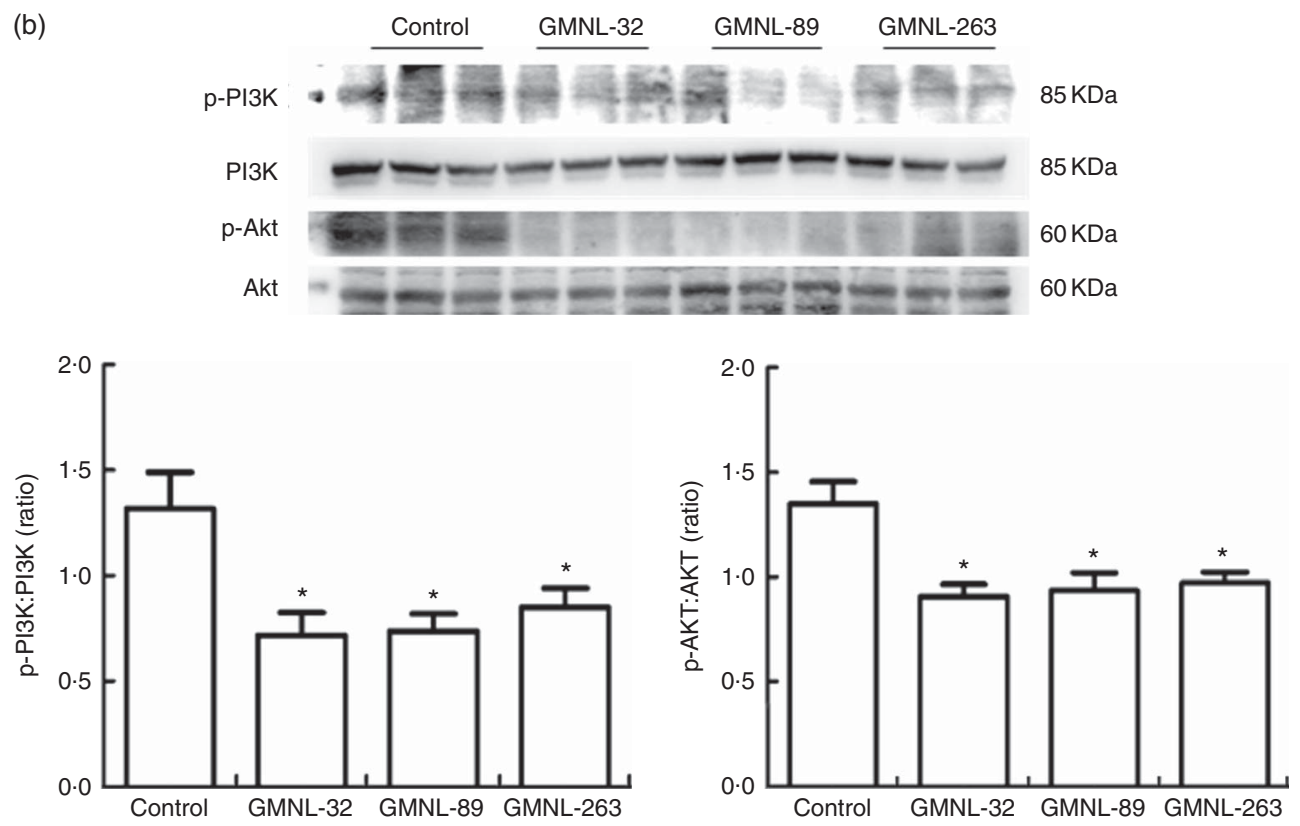

Fig. 4. Effects of Lactobacillus paracasei GMNL-32 (GMNL-32), Lactobacillus reuteri GMNL-89 (GMNL-89) and L. reuteri GMNL-263 (GMNL-263) on myeloid differentiation primary response gene 88 (MyD88), phosphoinositide 3-kinase (PI3K) and protein kinase B (AKT). Liver lysates obtained from NZB/W F1 mice receiving vehicle (control) ( $n$ 8), GMNL-32 ( $n$ 8), GMNL-89 $(n 8)$ or GMNL-263 $(n$ 8), respectively, were probed with antibodies against (a) MyD88, (b) phosphorylated PI3K (p-PI3K), PI3K, phosphorylated AKT (p-AKT), AKT. Bars represent the relative protein quantification of MyD88 on the basis of $\beta$-actin, p-PI3K on the basis of PI3K and $\mathrm{p}$-AKT on the basis of AKT. Similar results were observed in three repeated experiments. Mean value was significantly different from that of the control group: ${ }^{*} P<0.05$.

$\mathrm{T}$ cells in NZB/W F1 mice was detected. Fig. 5(a) presents representative results concerning the percentage of CD4+CD25+ $\mathrm{T}$ cells among all spleen cells, obtained by flow cytometry. No significant difference in the percentage of splenic CD4+CD25+ $\mathrm{T}$ cells was detected between NZB/W F1 mice that were treated with GMNL-32 or GMNL-89 and those that had been given the vehicle (control) (Fig. 5(a)). Notably, significantly more splenic CD4+CD25+ T cells were detected in NZB/W F1 mice that had been treated with GMNL-263 than in those that had been given the vehicle (control) (Fig. 5(a)). The mean percentages of splenic CD $4+\mathrm{CD} 25+\mathrm{T}$ cells for $\mathrm{NZB} / \mathrm{W}$ F1 mice that had been given the vehicle (control), GMNL-32, GMNL-89 and GMNL-263 were 1.99 (SD 0.77), 1.88 (SD 0.85), 1.94 (SD 0.68) and 2.63 (SD 0.95)\%, respectively. The number of CD 4+CD25+ $\mathrm{T}$ cells as a percentage of splenic CD4+ $\mathrm{T}$ cells in NZB/W F1 mice that had been treated with GMNL-263 was significantly higher than that in mice that had been given the vehicle (control) (Fig. 5(b)). A significantly higher mRNA level of Foxp3 was also detected in CD4+CD25+ $\mathrm{T}$ cells from NZB/W F1 mice that had been treated with GMNL-263 than in mice that had been given the vehicle (control) (Fig. 5(c)). 
(a)
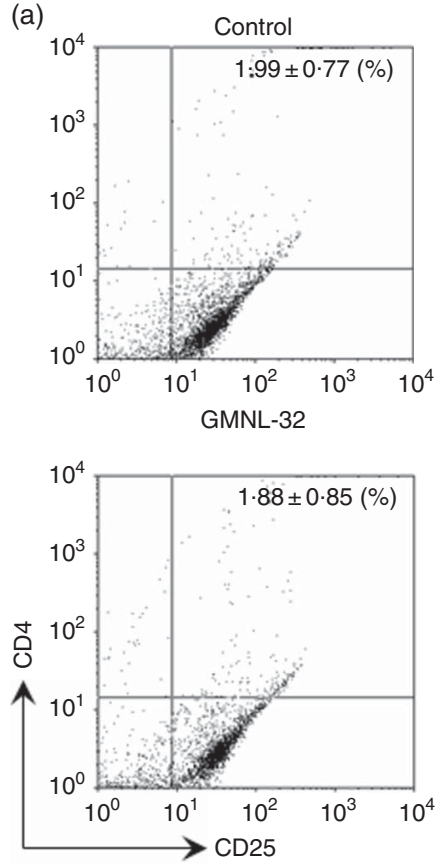

(b)

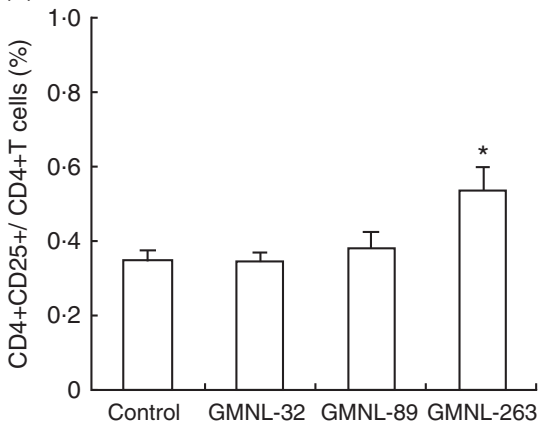

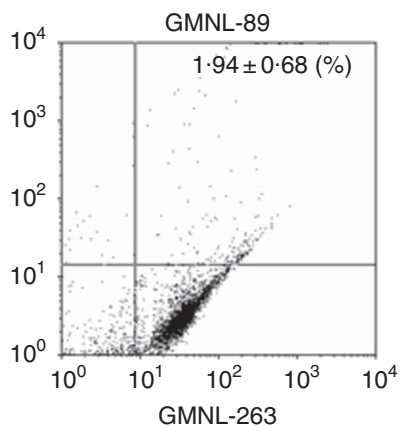

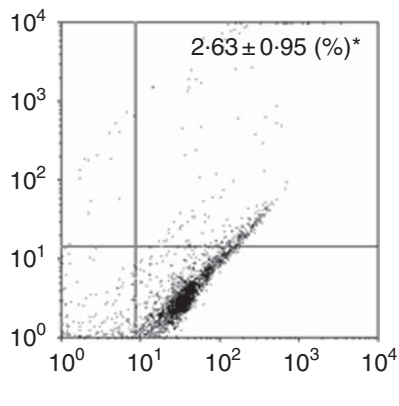

(c)

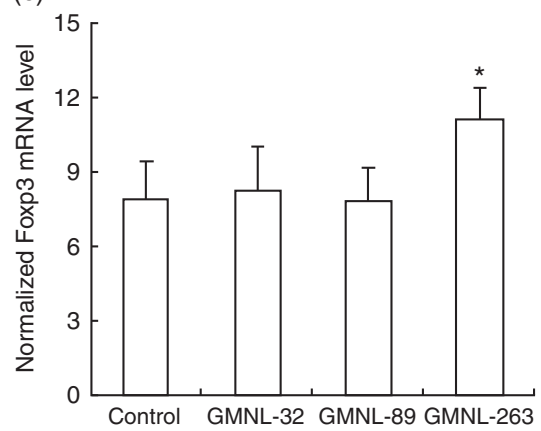

Fig. 5. Effects of Lactobacillus paracasei GMNL-32 (GMNL-32), Lactobacillus reuteri GMNL-83 (GMNL-83) and L. reuteri GMNL-263 (GMNL-263) on splenic CD4+/ CD25+/forkhead box P3 (Foxp3) regulatory T cell expression. (a) The relative percentage of splenic CD4+CD25+ regulatory T cells in NZB/W F1 mice were detected. The CD3+ $T$ cells were gated and the percentage of CD25+ cells in CD4+ T cells was shown. (b) The ratio of CD4+CD25+ T cells relative: CD4+ T cells and (c) Foxp3 mRNA level in CD4+CD25+ T cells were calculated. Values are means ( $n 8$ animals/group), and standard deviations represented by vertical bars. Similar results were observed in three repeated experiments. Mean value was significantly different from that of the control group: ${ }^{*} P<0.05$.

\section{Discussion}

Accumulating evidence reveals that probiotics have beneficial effects on human health and are effective therapeutic additions under clinical conditions ${ }^{(36)}$. Notably, certain Lactobacillus strains have been suggested to be beneficial in the treatment of various autoimmune disorders such as immune thyroid diseases and rheumatoid arthritis ${ }^{(37,38)}$. However, available information on the effects of probiotic bacteria in SLE is limited. The present study, for the first time, reports that supplementary GMNL-32, GMNL-89 and GMNL-263 significantly increase serum antioxidant activity in NZB/W F1 mice, reduce proinflammatory cytokine levels and weaken TLR/MyD88 signalling. Interestingly, NZB/W F1 mice that are treated with GMNL-263 exhibit significantly increased CD4+CD25+FoxP3+ T-cell expression. These findings suggest the potential of GMNL-32, GMNL-89 and GMNL-263 for use in comprehensive remedies for SLE patients.
Excessive oxidative stress and reduced antioxidant activities are commonly identified in both lupus-prone animals and patients with $\operatorname{SLE}^{(35,39,40)}$. The uncontrolled oxidative stress in SLE contributes to functional oxidative modifications of cellular protein, lipid and DNA, and oxidative modification has crucial consequences for immunomodulation and triggers autoimmunity $^{(7,41)}$. Previous studies have demonstrated that excess oxidative stress causes 8-oxodeoxyguanine and oxidised (LDL in SLE and leads to the generation of autoantibodies ${ }^{(7)}$. Therefore, the prevention of damage that would otherwise be caused by excessive oxidative stress is a very feasible strategy for treating SLE. In this study, supplementary GMNL-32, GMNL-89 or GMNL-263 significantly increased the antioxidant activities, including GSH and DPPH levels, in both serum and livers in NZB/W F1 mice. Significantly lower lipid oxidation such as reduced MDA level was detected in the serum and liver of $\mathrm{NZB} / \mathrm{W}$ F1 mice than in those of mice that were treated with 


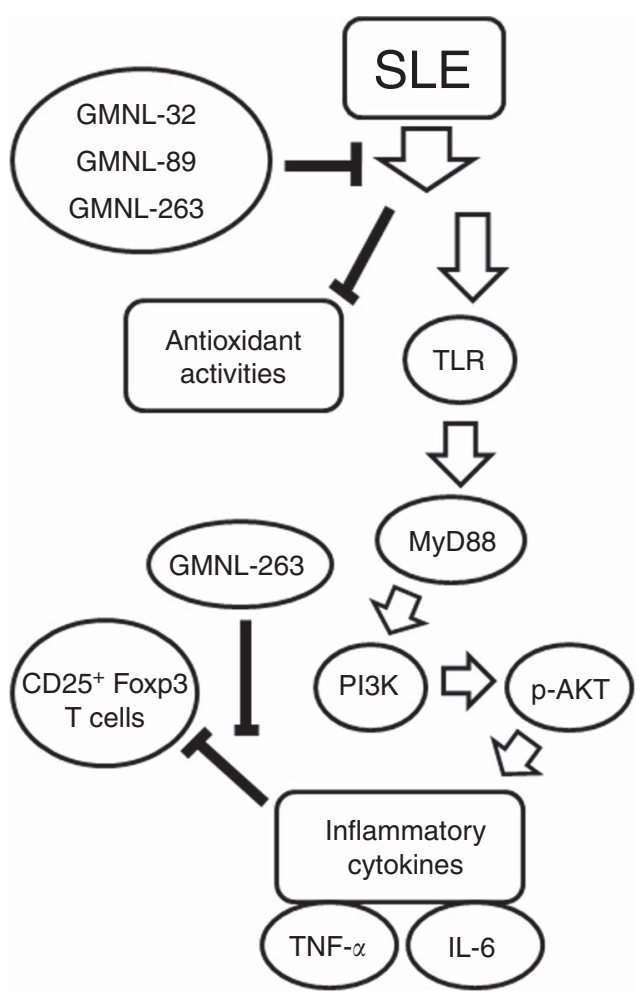

Fig. 6. Schematic illustration of possible mechanism of Lactobacillus bacterias involved in the pathogenesis of systemic lupus erythematosus (SLE). Lactobacillus reuteri GMNL-263 (GMNL-263) increase serum antioxidant activities and $\mathrm{CD} 4+\mathrm{CD} 25+$ regulatory $\mathrm{T}$ cells through inhibiting $\mathrm{PI} / 3 \mathrm{~K} / \mathrm{p}$ protein kinase B signalling in NZB/W F1 mice. GMNL-32, Lactobacillus paracasei GMNL-32; GMNL-89, Lactobacillus reuteri GMNL-89; TLR, toll-like receptors; MyD88, myeloid differentiation primary response gene 88; FOXp3, forkhead box P3; PI3K, phosphoinositide 3-kinase; p-AKT, phosphorylated protein kinase $\mathrm{B}$.

GMNL-32, GMNL-89 or GMNL-263. These findings suggest that GMNL-32, GMNL-89 and GMNL-263 reduce oxidative stress in cases of SLE.

TLR are crucial for host defense and have been implicated in the pathogenesis of autoimmune diseases by the recognition of self-molecules ${ }^{(42)}$. In SLE patients, autoreactive cells are expanded and activated, and they generate autoantibodies, especially against nuclear antigens. Therefore, TLR stimulation is suggested to contribute to the aggravation of the aberrant adaptive immune response in SLE patients ${ }^{(43)}$. Various TLR molecules, including TLR-2, TLR-3, TLR-4, TLR-5, TLR-7, TLR-8 and TLR-9, were shown to be involved in the pathogenesis of SLE in both experimental mouse models and clinical samples ${ }^{(42)}$. Therefore, these TLR elicited TLR/MyD88 signalling and induced the expression of proinflammatory cytokines, such as interferon- $\alpha / \beta$ (IFN- $\alpha / \beta)$, TNF- $\alpha$ and IL-6 (IL-6) in SLE ${ }^{(44)}$. Liver injuries have been suggested to be important for the generation of autoimmunity in $\operatorname{SLE}^{(45-48)}$. In this work, supplementary GMNL-32, GMNL-89 and GMNL-263 significantly reduced the expressions of TLR-4, TLR-5, TLR-7, TLR-9, MyD88, phosphorylated PI3K and AKT in the livers of NZB/W F1 mice. Although NZB/W F1 mice do not develop a detectable level of circulating IFN- $\alpha^{(49)}$, significantly reduced serum IL-6 and TNF- $\alpha$ levels were detected in NZB/W F1 mice that were treated with GMNL-32, GMNL-89 and GMNL-263. These findings support the potential involvement of GMNL-32, GMNL-89 and GMNL-263 in reducing TLR-mediated inflammation in the liver in cases of SLE, which may contribute to the amelioration of autoimmunity.

Regulatory T cells (CD4+CD25+FoxP3+) play pivotal roles in suppressing inflammation and maintaining the tolerance of immunity $^{(27)}$. An imbalance of regulatory T cells may lead to the generation of autoimmunity. Therefore, dysregulation of regulatory $\mathrm{T}$ cells in SLE has been associated with elevated oxidative stress and the irregular activation of Th1 cells ${ }^{(50,51)}$. A lower proportion of regular T cells in NZB/W F1 mice than in non-autoimmune mice was also observed ${ }^{(52)}$. Given the critical role of $\mathrm{CD} 4+\mathrm{CD} 25+\mathrm{FoxP} 3+\mathrm{T}$ cells in maintaining immune homoeostasis, the fact that many microbial species and their metabolites have the potential to induce CD4+CD25+FoxP3+ $\mathrm{T}$ cells is unsurprising ${ }^{(27)}$. Notably, the administration of GMNL-263 to NZB/W F1 mice significantly increases the percentage of their CD4+CD25+FoxP3+ T cells, suggesting that GMNL-263 reduces autoimmune phenomena in cases of SLE. However, the precise mechanism by which GMNL-263 induces CD4+CD25+FoxP3+ T cells in SLE requires further investigation.

Notably, only GMNL-263, but not GMNL-32 and GMNL-89, significantly induces the expression of splenic CD4+CD25+FoxP3 regulatory T cells in NZB/W F1 mice. This is probably because of their different properties. According to the analysis with API-ZYM system (API-Labs Ltd) reported by GenMont Biotech, Inc., Tainan, Taiwan, distinct patterns of enzyme production emerge in these Lactobacillus species. GMNL-32 exhibits the enzymatic activities of alkaline phosphatase, esterase (C4), esterase lipase (C8), lipase (C14), leucine arylamidase, valine arylamidase, cystine arylamidase, trypsin, $\alpha$-chymotrypsin, acid phosphatase, naphthol-AS-BIphosphohydrolase, $\alpha$-galactosidase, $\beta$-galactosidase, $\alpha$-glucosidase, $\beta$-glucosidase and $N$-acetyl- $\beta$-glucosaminidase. GMNL-89 exhibits the enzymatic activities of leucine arylamidase, acid phosphatase, naphthol-AS-BI-phosphohydrolase, $\alpha$-galactosidase, $\beta$-galactosidase and $\alpha$-glucosidase. GMNL-263 exhibits the enzymatic activities of esterase lipase (8), leucine arylamidase, valine arylamidase, acid phosphatase, naphthol-AS-BI-phosphohydrolase, $\alpha$-galactosidase, $\beta$-galactosidase and $\alpha$-glucosidase. Moreover, biochemical tests were also performed by using API50 $\mathrm{CH}$ (BioMerieux). GMNL-32 utilised D-mannitol, D-sorbitol, methyl- $\alpha$-D-glucopyranoside, D-ribose, $N$-acetyl glucosamine, gentiobiose, amygdalin, D-turanose, arbutin, esculin ferric citrate, D-tagatose, Salicin, D-galactose, D-cellobiose, D-glucose, D-maltose, D-fructose, D-mannose, potassium gluconate, L-sorbose, D-saccharose (sucrose), D-trehalose and D-melezitose as a sole carbon source. GMNL-89 utilised D-raffinose, L-arabinose, D-ribose, esculin ferric citrate, D-galactose, D-glucose, D-maltose, D-lactose (bovine origin), D-melibiose, Potassium gluconate and D-saccharose (sucrose) as a sole carbon source. Notably, GMNL-263 only utilised D-raffinose, L-arabinose, D-galactose, D-glucose, D-maltose, D-melibiose, potassium gluconate and D-saccharose (sucrose) as a sole carbon source. These findings indicated the different properties among GMNL-32, GMNL-89 and GMNL-263. Although further investigations are required to verify the precise roles of these differences on immune modulation, this study at least provides a possible explanation 
that GMNL-263-increased splenic CD4+CD25+FoxP3 regulatory $\mathrm{T}$ cells in NZB/W F1 mice may be because of to its different enzyme pattern and biochemical properties as compared with those of GMNL-32 and GMNL-89.

Our recent publication has reported that supplementation with GMNL-32, GMNL-89 or GMNL-263 in NZB/W F1 mice reduces the expressions of hepatic IL- $1 \beta$, IL- 6 and TNF- $\alpha$ proteins by suppressing the MAPK (extracellular signal-regulated kinases, P38 and c-Jun N-terminal kinase) and IкB kinase/ $\mathrm{NF}-\kappa \mathrm{B}$ signalling pathways ${ }^{(33)}$. Fig. 6 in this study further reveals that supplementary GMNL-32, GMNL-89 and GMNL-263 significantly increase the serum and liver levels of antioxidant enzymes such as GSH and DPPH in NZB/W F1 mice and reduce the levels of oxidative lipids such as MDA and inflammatory cytokines, such as TNF- $\alpha$ and IL-6. Moreover, GMNL-32, GMNL-89 and GMNL-263 significantly reduce innate-immunitybased inflammatory signalling, TLR/MyD88 signalling, in the livers of NZB/W F1 mice. Notably, supplementation with GMNL-263 significantly increases the population of splenic $\mathrm{CD} 4+\mathrm{CD} 25+\mathrm{FoxP} 3$ regulatory $\mathrm{T}$ cells in NZB/W F1 mice.

\section{Conclusions}

This study proposes that, besides the anti-oxidative stress and anti-inflammation effects of GMNL-32, GMNL-89 and GMNL-263, the induction of CD4+CD25+FoxP3+ T cells by GMNL-263 could lead to the development of a new therapeutic approach to curbing the autoimmune response in cases of SLE, providing an alternative to detrimental immunosuppressive remedies.

\section{Acknowledgements}

This work was supported by GenMont Biotech, Inc., Tainan, Taiwan (E0150100). GenMont Biotech had no role in the design, analysis or writing of this article.

T.-C. H., B.-S. T. and C.-Y. H. conceived and designed the experiments; T.-C. H., C.-H. L., K.-C. H., Y.-H. C. and B.-S. T. performed the experiments and analysed the data; and T.-C. H. and B.-S. T. wrote the paper.

The authors declare that there are no conflicts of interest.

\section{References}

1. Gerritsen J, Smidt H, Rijkers GT, et al. (2011) Intestinal microbiota in human health and disease: the impact of probiotics. Genes Nutr 6, 209-240.

2. Wang G, Cai P, Ansari GA, et al. (2007) Oxidative and nitrosative stress in trichloroethenemediated autoimmune response. Toxicology 229, 186-193.

3. Wang G, Pierangeli SS, Papalardo E, et al. (2010) Markers of oxidative and nitrosative stress in systemic lupus erythematosus: correlation with disease activity. Arthritis Rheum 62, 2064-2072.

4. Ozkan Y, Yardým-Akaydýn S, Sepici A, et al. (2007) Oxidative status in rheumatoid arthritis. Clin Rheumatol 26, 64-68.

5. Grimsrud PA, Xie H, Griffin TJ, et al. (2008) Oxidative stress and covalent modification of protein with bioactive aldehydes. J Biol Chem 283, 21837-21841.
6. Frostegård J, Svenungsson E, Wu R, et al. (2005) Lipid peroxidation is enhanced in patients with systemic lupus erythematosus and is associated with arterial and renal disease manifestations. Arthritis Rheum 52, 192-200.

7. Kurien BT \& Scofield RH (2008) Autoimmunity and oxidatively modified autoantigens. Autoimmun Rev 7, 567-573.

8. Jacob N \& Stohl W (2011) Cytokine disturbances in systemic lupus erythematosus. Arthritis Res Ther 13, 228.

9. Hahn BH (1993) An overview of the pathogenesis of systemic lupus erythematosus. In Dubois' Lupus Erythematosus, pp. 69-76 [DJ Wallace and BH Hahn, editors]. Philadelphia, PA: Williams and Wilkins.

10. Linker-Israeli M, Deans RJ, Wallace DJ, et al. (1991) Elevated levels of endogenous IL-6 in systemic lupus erythematosus. A putative role in pathogenesis. J Immunol 147, 117-123.

11. Peterson E, Robertson AD \& Emlen W (1996) Serum and urinary interleukin-6 in systemic lupus erythematosus. Lupus 5, 571-575.

12. Schiffer L, Bethunaickan R, Ramanujam M, et al. (0082) Activated renal macrophages are markers of disease onset and disease remission in lupus nephritis. J Immunol 180, 1938-1947.

13. Cash H, Relle M, Menke J, et al. (2010) Interleukin 6 (IL-6) deficiency delays lupus nephritis in MRLFaslpr mice: the IL-6 pathway as a new therapeutic target in treatment of autoimmune kidney disease in systemic lupus erythematosus. J Rheumatol 37, 60-70.

14. Gröndal G, Gunnarsson I, Rönnelid J, et al. (2000) Cytokine production, serum levels and disease activity in systemic lupus erythematosus. Clin Exp Rheumatol 18, 565-570.

15. Boswell JM, Yui MA, Burt DW, et al. (1988) Increased tumor necrosis factor and IL-1 beta gene expression in the kidneys of mice with lupus nephritis. J Immunol 141, 3050-3054.

16. Yokoyama H, Kreft B \& Kelley VR (1995) Biphasic increase in circulating and renal TNF- $\alpha$ in MRL-lpr mice with diff ering regulatory mechanisms. Kidney Int 47, 122-130.

17. Studnicka-Benke A, Steiner G, Petera P, et al. (1996) Tumour necrosis factor alpha and its soluble receptors parallel clinical disease and autoimmune activity in systemic lupus erythematosus. BrJ Rheumatol 35, 1067-1074.

18. Gabay C, Cakir N, Moral F, et al. (1997) Circulating levels of tumor necrosis factor soluble receptors in systemic lupus erythematosus are signifi cantly higher than in other rheumatic diseases and correlate with disease activity. J Rheumatol 24, 303-308.

19. Lescheid DW (2014) Probiotics as regulators of inflammation: a review. Funct Foods Health Dis 4, 299-311.

20. de Moreno de LeBlanc A, Matar C \& Perdigon G (2007) The application of probiotics in cancer. Br J Nutr 98, Suppl. 1, S105-S110.

21. Laitinen K, Poussa T \& Isolauri E (2009) Probiotics and dietary counselling contribute to glucose regulation during and after pregnancy: a randomised controlled trial. Br J Nutr $\mathbf{1 0 1}$, 1679-1687.

22. Thomas CM \& Versalovic J (2010) Probiotics-host communication. Modulation of signaling pathways in the intestine. Gut Microbes 1, 148-163.

23. Bermudez-Brito M, Plaza-Diaz J, Munoz-Quezada S, et al. (2012) Probiotic mechanisms of action. Ann Nutr Metab 61, $160-174$

24. Di Giacinto CD, Marinaro M, Sanchez M, et al. (2005) Probiotics ameliorate recurrent Th- 1 mediated murine colitis by inducing IL-10 and IL-10-dependent TGF- $\beta$ bearing regulatory cells. J Immunol 174, 3237-3246.

25. Timmerman HM, Koning CJ, Mukder L, et al. (2004) Monostrain, multistrain and multispecies probiotics - a comparison of functionality and efficacy. Int J Food Microbiol 96, 219-233. 
26. Honad K \& Littman DR (2012) The microbiome in infectious disease and inflammation. Ann Rev Immunol 30, 758-795.

27. Dwivedi M, Kumar P, Laddha NC, et al. (2016) Induction of regulatory $\mathrm{T}$ cells: a role for probiotics and prebiotics to suppress autoimmunity. Autoimmun Rev 15, 379-392.

28. Makarova K, Slesarev A, Wolf Y, et al. (2006) Comparative genomics of the lactic acid bacteria. Proc Natl Acad Sci US A 103, 15611-15616.

29. Goldstein EJ, Tyrrell KL \& Citron DM (2015) Lactobacillus species: taxonomic complexity and controversial susceptibilities. Clin Infect Dis 60, S98-S107.

30. Liao PH, Kuo WW, Hsieh DJ, et al. (2016) Heat-killed Lactobacillus reuteri GMNL-263 prevents epididymal fat accumulation and cardiac injury in high-calorie diet-fed rats. Int J Med Sci 13, 569-577.

31. Ting WJ, Kuo WW, Hsieh DJ, et al. (2015) Heat killed Lactobacillus reuteri GMNL-263 reduces fibrosis effects on the liver and heart in high fat diet-hamsters via TGF- $\beta$ suppression. Int J Mol Sci 16, 25881-25896.

32. Tian XP \& Zhang X (2010) Gastrointestinal involvement in systemic lupus erythematosus: insight into pathogenesis, diagnosis and treatment. World $J$ Gastroenterol 16, 2971-2977.

33. Hsu TC, Huang CY, Liu CH, et al. (2017) Lactobacillus paracasei GMNL-32, Lactobacillus reuteri GMNL-89 and L. reuteri GMNL-263 ameliorate hepatic injuries in lupus-prone mice. Br J Nutr 117, 1066-1074.

34. Lin CH, Lin CC, Shibu MA, et al. (2014) Oral Lactobacillus reuteri GMN-32 treatment reduces blood glucose concentrations and promotes cardiac function in rats with streptozotocininduced diabetes mellitus. Br J Nutr 111, 598-605.

35. Hsu TC, Chiu CC, Wang YW, et al. (2013) Effects of cystamine on antioxidant activities and regulatory $\mathrm{T}$ cells in lupusprone mice. J Cell Mol Med 17, 1308-1315.

36. Isolauri E (2001) Probiotics in human disease. Am J Clin Nutr 73, 1142S-1146S

37. Schiffer C, Lalanne AI, Cassard L, et al. (2011) A strain of Lactobacillus casei inhibits the effector phase of immune inflammation. J Immunol 187, 2646-2655.

38. Zamani B, Golkar HR, Farshbaf S, et al. (2016) Clinical and metabolic response to probiotic supplementation in patients with rheumatoid arthritis: a randomized, double-blind, placebo-controlled trial. Int J Rheum Dis 19, 869-879.
39. Morgan PE, Sturgess AD \& Davies MJ (2005) Increased levels of serum protein oxidation and correlation with disease activity in systemic lupus erythematosus. Arthritis Rheum $\mathbf{5 2}$, 2069-2079.

40. Shah D, Mahajan N, Sah S, et al. (2014) Oxidative stress and its biomarkers in systemic lupus erythematosus. J Biomed Sci 21, 23.

41. Perl A (2013) Oxidative stress in the pathology and treatment of systemic lupus erythematosus. Nat Rev Rheumatol 9, 674-686.

42. Wu YW, Tang W \& Zuo JP (2015) Toll-like receptors: potential targets for lupus treatment. Acta Pharmacol Sin 36, 1395-1407.

43. Celhar T, Magalhães R \& Fairhurst AM (2012) TLR7 and TLR9 in SLE: when sensing self goes wrong. Immunol Res 53, 58-77.

44. Lyn-Cook BD, Xie C, Oates J, et al. (2014) Increased expression of Toll-like receptors (TLRs) 7 and 9 and other cytokines in systemic lupus erythematosus (SLE) patients: ethnic differences and potential new targets for therapeutic drugs. Mol Immunol 61, 38-43.

45. Hsu TC, Chiang SY, Wu JH, et al. (2008) Treatment with taurine attenuates hepatic apoptosis in NZB/W F1 mice fed with a high-cholesterol diet. J Agric Food Chem 56, 9685-9691.

46. Hsu TC, Huang CY, Chiang SY, et al. (2008) Transglutaminase inhibitor cystamine alleviates the abnormality in liver from NZB/W F1 mice. Eur J Pharmacol 579, 382-389.

47. Tzang BS, Chiang SY, Lai WX, et al. (2008) Treatment with cystamine reduces apoptosis in liver from NZB/W F1 mice. Int Immunopharmacol 8, 589-596.

48. Hsu TC, Chen YC, Tsai CC, et al. (2010) Protective effects of taurine against hepatic abnormality in NZB/W F1 mice fed a hypercholesterolemic diet. Food Chem 119, 62-68.

49. Mathian A, Weinberg A, Gallegos M, et al. (2005) IFN-alpha induces early lethal lupus in preautoimmune (New Zealand Black $\mathrm{x}$ New Zealand White) $\mathrm{F} 1$ but not in BALB/c mice. J Immunol 174, 2499-2506.

50. Wang G, König R, Ansari GA, et al. (2008) Lipid peroxidationderived aldehyde-protein adducts contribute to trichloroethene mediated autoimmunity via activation of CD4T cells. Free Radic Biol Med 44, 1475-1482.

51. La Cava A (2008) T-regulatory cells in systemic lupus erythematosus. Lupus 17, 421-425.

52. Chen Y, Cuda C \& Morel L (2005) Genetic determination of T cell help in loss of tolerance to nuclear antigens. J Immunol 174, 7692-7702. 